\title{
Recognising outstanding care
}

\author{
Emeritus Professor Alan Glasper, from the University of Southampton, discusses a report from the Care \\ Quality Commission that sets out some examples of good and outstanding care
}

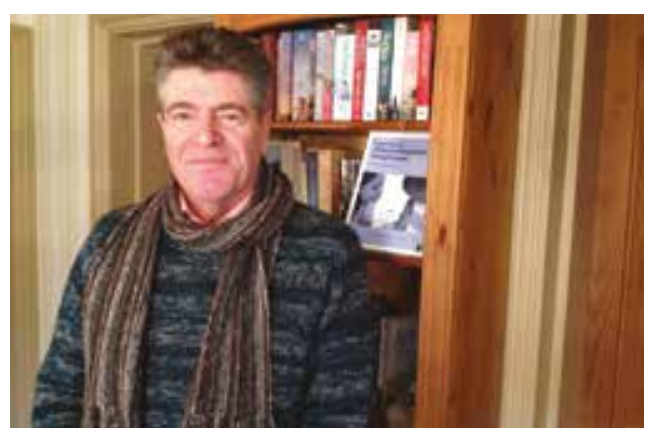

I n April 2017 the health and social care regulator for England, the Care Quality Commission (CQC), published Celebrating Good Care, Championing Outstanding Care, a report that gives credit to healthcare services that have demonstrably delivered good and outstanding care to service users, thus recognising where care is good and celebrating the achievements of those who work in institutions that are getting it right (CQC, 2017).

The CQC had reported on the care delivery profile of health services across England in its 'State of Care' report, published in late 2016, which showed that there was considerable variety in the quality of care provided to service users (CQC, 2016). It revealed that the best care was in services that acknowledged that service delivery improvement is a continuous quest and where staff were proactive in seeking feedback on their services and learning from any mistakes.

Having completed the 2015 to 2016 inspections, the CQC now has a corporate vision of what healthcare delivery looks like within organisations throughout England. The regulator is confident that the data it has obtained on national care provision will foster a learning culture through which lessons can be learned. The publication of its latest report (CQC, 2017a) is purposely focused on celebrating the good and outstanding care that the CQC inspectors and their specialist advisers have witnessed during their inspections. The CQC was keen to highlight those organisations that reach or exceed its expectations of what a good service should look like. This is because some healthcare facilities address the CQC measurable domains through innovative new ways of working, or by meeting basic aspects of care delivery well. The CQC believes that similar organisations can learn from these high achievers and that their solutions to fully meeting the CQC standards should be shared with others across the healthcare industry.

\section{Background}

The CQC carries out periodic inspections of hospitals and other care facilities, sometimes unannounced. During these inspections CQC inspectors are joined by teams of specialist advisers from each of the core services being inspected. Hence in the case of surgery, the inspector for that core service will have the assistance and advice of staff from other institutions, such as a senior surgeon, a specialist surgical nurse and an anaesthetist. This team will examine all aspects of surgery to ensure that the service is compliant with current practice and policy pertinent to the care of the patients. For example, during the inspection of surgical services, the specialist advisers will endeavour to witness the World Health Organization (WHO) Safe Surgery Checklist being used. This checklist identifies three phases of an operation, and a checklist coordinator must confirm that the surgery team has completed all listed tasks in a phase before it proceeds to the next stage (World Alliance for Patient Safety, 2008).

The CQC inspection teams are instructed to examine health care in five domains: 'safe', 'effective', 'caring', 'responsive' and 'well led'.

For each of these domains the inspection teams will follow a range of key lines of enquiry that provide prompts to help the teams make judgements about the core service being inspected. Hence in the case of children's and young people's services, under the domain of 'safe', the team members will expect to see that deterioration in a sick child's condition is assessed and monitored using the Royal College of Paediatrics and Child Health (RCPCH) Situation Awareness for Everyone (SAFE) Programme (RCPCH, 2016). Among other aims, this seeks to improve communication between all health professionals involved in a child's care to ensure treatment is consistent in protecting sick children from potential harms caused by unrecognised clinical deterioration (Glasper, 2016; RCPCH, 2016).

In conjunction with data gathered from observations and discussions with members of staff of all grades and from patients and their relatives, CQC inspection teams also have access to data provided by the hospital or other facility under the statutory CQC intelligent monitoring process. This gives inspectors and their teams a clearer picture of areas that may need to be followed up during an inspection.

When all of the data have been analysed the final objective decisions about a core service and the institution as a whole are made. Using this process each core service is awarded a grade, ranging from 'outstanding', 'good', 'requires improvement' to 'inadequate'. The aggregation of these grades under the five domains gives a collective corporate grade for the institution in question. Even within domains that are graded 'inadequate' it is possible for some core services to be 'outstanding' or 'good'. When grades of 'requires improvement' or 'inadequate' are made the CQC has statutory powers to ensure that action plans are formulated to address them, including the application of special measures, a process designed to ensure there is a timely and coordinated response where the standard of care has been judged to be inadequate (CQC, 2017b).

For example, in April 2017, the CQC recommended a return to special measures for the United Lincolnshire Hospitals NHS Trust and, as part of that process, stated that, in particular, there must be significant improvements in the quality and safety of patient care (CQC, 2017c).

This latest CQC report is, however, designed to celebrate good and outstanding care delivery and seeks to publicly 
acknowledge the hard effort of many, including nurses, to push forward the frontiers of optimum health care for patients.

\section{Celebrating outstanding care}

The CQC's primary mission is to drive change that will improve the quality of care that patients receive as service users. In identifying excellence and showcasing how some providers have addressed complex care challenges, it hopes to inspire others to emulate their successes. The report showcases a number of examples of how institutions have succeeded in achieving high grades in the inspection domains, some of which are highlighted below (CQC, 2017a).

\section{Safe}

The Southdene Medical Centre at Shotton Colliery in County Durham achieved an 'outstanding' rating in three out of the CQC's five key domains. The leadership's focus on safety was of high quality. In particular, the inspection team saw that patients were protected by robust safety systems and the inspectors witnessed active learning among the staff following the reporting of safety incidents. In monitoring and learning from these incidents, the medical practice used innovative and proactive methods to improve patient outcomes and hence the outcomes for patients were noted to be consistently better at Southdene than for those who use similar services elsewhere. Patients were prioritised and protected from harm and any significant adverse events were discussed at practice team meetings, which were minuted and formed part of an annual review that was available for inspection. For example, after incidents related to patients' post-hospital follow-up appointments were identified, action was taken to ensure that a monitoring process was put in place. Southdene's strengths within the 'safe' domain were underpinned by its leadership team's quest for continuous improvement (CQC, 2017a).

\section{Effective}

The report identified University Hospitals Bristol NHS Foundation Trust as being 'outstanding' under the effectiveness domain.

I Only 2 years previously this trust had been rated as 'requires improvement' and it was the first hospital trust in England to move from 'requires improvement' to 'outstanding' between two inspections. The CQC report shows that the trust is now one of the highest-rated trusts in the country and it attributes this achievement to the development of a culture of continuous improvement. In addition to 'effective', the trust was also rated 'outstanding' in the 'well-led' domain but it was the truly holistic approach to planning and people's discharge or transfer to other services that contributed to the trust's outstanding effectiveness in providing care for its patients. The CQC inspectors noted that staff worked collaboratively in this process to understand the range and complexity of people's needs and undertook comprehensive patient assessments, including of their mental and physical health and wellbeing, and nutrition and hydration needs (CQC, 2017a).

\section{Caring}

Birmingham Children's Hospital NHS

Foundation Trust was the first specialist children's hospital to achieve an 'outstanding' rating from CQC. It was awarded this grade in the caring, effective and responsive domains. The inspection team noted that, in addition to helping ward staff with care delivery, clinical nurse specialists also provided additional emotional support for children and families within different medical specialties. Preoperative specialist support for patients and their relatives was available to families before surgery and parents could stay overnight in nearby parental accommodation free of charge. This facility also gave parents the opportunity of meeting other families in similar situations, thus providing additional supportive strategies. The CQC report showed that the hospital had made significant differences to the lives of the children, young people and parents who used the service.

\section{Responsive}

The CQC report identified that the Northumbria Healthcare NHS Foundation Trust specialist community mental health services for children and young people had been awarded 'outstanding' for the 'responsive' domain. Staff were described as being passionate, enthusiastic and dedicated to their work with children and young people. Young service users and their family members spoke very positively to the CQC inspectors about the staff who worked there (CQC, 2017a).

\section{Well led}

The CQC report identified that Harley House Care Home in Leicester, a facility that

\section{KEY POINTS}

- In April 2017 the Care Quality Commission (CQC) published a report focused on celebrating care rated as 'good' and 'outstanding'

- Some healthcare facilities address the CQC's measurable standards through innovative new ways of working, or by meeting basics aspects of care delivery well

- The CQC believes that healthcare institutions can learn from the examples of high achievers and that their solutions to fully meeting the CQC standards should be shared with others

provides personal care support, including nursing care, for up to 28 people, was exceptionally well led. It achieved the 'outstanding' rating in this domain because it was perceived by the inspectors to be exemplary in responding to people's needs. The feedback the CQC obtained from service users and their family members led the inspectors to rate Harley House as 'outstanding' for its leadership, its responsiveness and caring (CQC, 2017).

\section{Conclusion}

The CQC report (CQC, 2017a) publicly acknowledges the contribution of various health professionals to maintaining and enhancing care delivery in many parts of the health service, often in difficult circumstances attributable to a time of austerity in the overall health economy. Many nurses will find this report inspiring and some may be motivated to emulate the examples of the successes described. BJN

Care Quality Commission (2016) The State of Health Care and Adult Social Care in England 2015/16. http://tinyurl. com/jfyp9f3 (accessed 24 May 2017)

Care Quality Commission (2017a) Celebrating Good Care, Championing Outstanding Care. http://tinyurl.com/ ksk6qz9 (accessed 24 May 2017)

Care Quality Commission (2017b) Special measures. http://tinyurl.com/z9ms5zy (accessed 24 May 2017

Care Quality Commission (2017c) CQC recommends a return to special measures for United Hospitals Lincolnshire NHS Trust. http://tinyurl.com/18onhv3 (accessed 24 May 2017)

Glasper A (2016) Keeping sick children in hospital SAFE from harm. Br J Nurs 25(20): 1044-5. https://doi. org/10.12968/bjon.2016.25.20.1144

Royal College of Paediatrics and Child Health (2016) Situation Awareness for Everyone (SAFE) Programme. http://tinyurl.com/nd3jmll (accessed 24 May 2017)

World Alliance for Patient Safety for the World Health Organization (2008) Surgical Safety Checklist. http:// tinyurl.com/7swy88x (accessed 24 May 2017) 